\title{
Adaptive based Sequential Implementation Resampling Particle Filter (ASIRPF) for Real Time Geophysical Applications
}

\author{
Wan Mohd Yaakob Wan Bejuri ${ }^{1}$, Mohd Murtadha Mohamad ${ }^{2}$, Siti Azirah Asmai ${ }^{1}$ and Rabiah Ahmad $^{1}$ \\ ${ }^{1}$ Centre for Advanced Computing Technology, Fakulti Teknologi Maklumat dan Komunikasi, \\ Universiti Teknikal Malaysia Melaka, MALAYSIA. \\ ${ }^{2}$ School of Computing, \\ Universiti Teknologi Malaysia, MALAYSIA. \\ yaakob@utem.edu.my
}

\begin{abstract}
The services of geophysical application in the obstructed area can be extremely challenging, especially if the Global Navigation Satellite System (GNSS) signal is unavailable or weak. Usually, the integration with the inertial sensor in the smartphone was used for assisting better navigation. Nonetheless, the usage of particle filter module in optimizing data from positioning sensor will contribute to the low particle sample size or known as sample impoverishment phenomenon, and finally increasing the location positioning error in a certain period. Adaptations towards to particle sample size and noise, must be made, to make the particle filter more intelligent, reliable and robust in a long time it is running. In this paper, we propose a new algorithm of sequential implementation resampling particle filter by adapting the particle sample size and sensor noise measurement. This adaptation will be used to counteract in a different situation. As the results, the paper shows the proposed solution can achieve an average improvement of $24.78 \%$ by reducing RMSE of state estimation compared to previous algorithm. In the future, it is expected to contribute to the modernization of geophysical applications.
\end{abstract}

Key words : Resampling, Sample Impoverishment, Inertial Navigation Systems, Global Navigation Satellite Systems.

\section{INTRODUCTION}

The signal quality of the GNSS is very crucial in the location positioning of geophysical applications. It can be caused by a different factor. The factor such as unavailability of GNSS measurements in the obstructed area, it is can make the signal unavailable or weak. Other than that, the characteristics of the GNSS antenna that shared with other communication systems (such as WiFi and Bluetooth) also, causing reducing the quality of GNSS measurements. All of these factors can cause inaccurate positioning result. Thus, the integration of GNSS with other sensor is needed to ensure the positioning result is better in term of accuracy and consistency. One of the major smartphone components, which is known as the inertial sensor, can be used to integrate with GNSS for assisting the navigation during unavailability or weak GNSS signal. It is can help to navigate the object in a dense environment such as in the building corridor. Apart from that, it has the capability to determine location anywhere without depends on building infrastructure since it can be found in many smartphones [1]. The example application of this sensor is mobile first-responder navigation and mobile emergency rescue and tracking [2][3][4][5]. Despite that, the major issues of geophysical application is sample impoverishment during particle filtering. This phenomenon causing insufficiency of particle sample size. Thus, it will increase the location positioning error in a certain period [6]. To counteract the phenomenon, an adaptation towards sequential implementation resampling particle filter can be used. To reduce positioning error in different environments. As real time geophysical applications need to work with different kinds of sensors and a varying number of particles, it would be beneficial if it could survive within different environments. Thus, we are proposing a new sequential implementation resampling particle filter by adapting noise and particle measurement. This adaptation is to determine the most suitable algorithm out of the sequential implementation resampling algorithms that could be used. To describe more detail, we divide the 
paper into nine sections. The first section is describing the introduction of the paper. Then, the second section is to describe the basic concept related to real time geophysical application. After that, in section three, we describe the problem formulation of this paper. Next, in section four, we present the aim of this research. After that, in section five, we present the proposed method for this paper. Then, in Section 6, we present more detail in term of the algorithm that will be used and manipulated in the proposed method. After that, we will present the experimental results in Section 7. Then, we conclude with a discussion and recommendations for further research in Section 8. Finally, we presenting reference in Section 9.

\section{ConcePt OF REAL TIME GEOPHYSICAL APPLICATION}

The usage of GNSS measurement signal for describing the earth phenomena has already been carried out in the field of seismology, meteorology and ionosphere. To do that, it requires dense information. In the case of a geodetic class or low-cost receivers such as, embedded GNSS smartphone, a densification of the actual network is provided through GSM network. According to the worldwide trend, smartphones is very popular among geophysical application user.

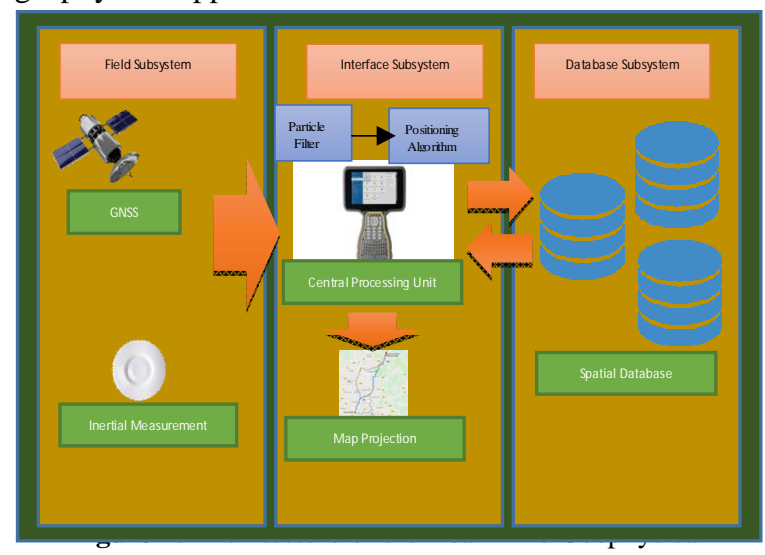

Application

It is can be considered a perfect solution to fulfil the requirements for GNSS data usage in geophysical application systems. The use of GNSS signal from different constellations especially from other geo satellite system such as BeiDou and Galileo, the accuracy of the location positioning could be increased. This is necessary when smartphones are used in geophysics because multipath and interference with other signal sources are projected into the reconstruction of the phenomenon of interest, reducing the reliability of the final solution. Despite that, most of the geophysical application developer preferring assisted inertial sensor integration, which is embedded in the smartphone, that can be used to integrate with GPS signal and can help to increase the location positioning also. This is considered a popular technique since it is embedded in many smartphones nowadays. In this section, we discuss the basic concept of real time geophysical applications. The concept is basically has been applied in many mobile application systems (see Figure 1 for fundamental system architecture) [7][8][9][10]. In order to applied it, it is requires multi sensor technique by fusing the standalone technique with other signal. [11][12][13] By using this concept, it may be enhanced using three dimensional (3D) mapping, context awareness and cooperation between users. According to Figure 1, the basic geophysical applications generally consist of three (3) subsystems which are, field subsystem, interface subsystem, and database subsystem. In a normal situation, the GPS sensor and inertial sensor will continuously send its signal to the central processing unit (which is located in the interface subsystem). The received signals will be processed by a central processing unit before it is compared with the spatial data in the database server. In the central processing unit, the particle filter and positioning algorithm are installed, where it is responsible to optimize the input signal and calculate positioning output. Finally, the system will display the output in form of a graphical map. Next, we will describe the research problem that will be highlighted in this paper.

\section{ReSEARCh Problem}

Generally, the purpose of the particle filter is to optimize the particle or data obtained from the sensor. In real time data transmission, the phenomenon so called sample impoverishment could occur. It is caused when a low number of particle transmitted during the particle filtering process [14]. This could make the optimization process of particle filtering will generate false positive result from real data.

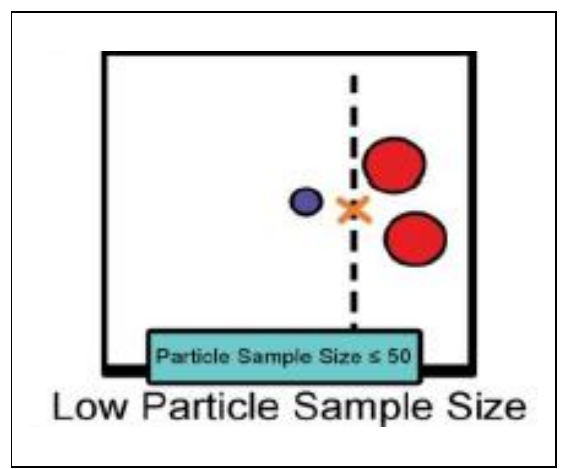

Figure 2: Illustration of Sample Impoverishment Phenomenon in Real Time Geophysical Application

Figure 2, shows the illustration of sample impoverishment phenomenon in real time geophysical application. It is also addressed the effect of data or particle transmitted through real time system, which is the number of transmitted data (which in particle form) 
become less. These situations depend on real time data transmission setup. Currently, there are a variety of sequential implementation resampling particle filter algorithm was used to counteract this phenomenon (refer to Table 1). If it used in a different situation, the filtered data will be not optimized, then, the state estimation error will suddenly be increased and finally will increase the location positioning error. There are three common types of sequential implementation resampling particle filter algorithm. First, the single distribution resampling particle filter. In this algorithm, it can do straight forward resampling only during stage of very low sample impoverishment. Second, the compound resampling particle filter. In this algorithm, it is can do low weighted particle resampling during the stage of low sample impoverishment, and finally or thirdly, the special strategies resampling particle filter can do modification only during stage of high sample impoverishment.

Table 1: Sample Impoverishment Situation

\begin{tabular}{|l|c|}
\hline $\begin{array}{l}\text { Sample } \\
\text { Impoverishment } \\
\text { Situation }\end{array}$ & Parameter Situation \\
\hline $\begin{array}{l}\text { Very low sample } \\
\text { impoverishment }\end{array}$ & $\begin{array}{r}\text { Noise measurement }(\mathrm{R})>0.1 \text { and Particle } \\
\text { Sample Size }(\mathrm{N})>100\end{array}$ \\
\hline $\begin{array}{l}\text { Low sample } \\
\text { impoverishment }\end{array}$ & $\begin{array}{r}\text { Noise measurement }(\mathrm{R})>0.1 \text { and Particle } \\
\text { Sample Size }(\mathrm{N}) \text { is } 81 \text { until 100 }\end{array}$ \\
\hline $\begin{array}{l}\text { High sample } \\
\text { impoverishment }\end{array}$ & $\begin{array}{r}\text { Noise measurement }(\mathrm{R}) \leq 0.1 \text { and Particle } \\
\text { Sample Size }(\mathrm{N}) \leq 80\end{array}$ \\
\hline
\end{tabular}

However, these algorithms are only suited to particular environments. If the situation changed, the state estimation error will be suddenly increased and finally, it will increase the positioning error of geophysical application. Thus, it would be useful if the algorithm were robust enough to survive in different situations.

\section{RESEARCH OBJECTIVE}

This study highlights the new design of sequential implementation resampling particle filter algorithm. In this proposed algorithm, it is adapts the noise measurement integrated with particle number before function. The proposed algorithm can be used to overcome the problem of sample impoverishment in different environments [15][16]. The following section will discuss the proposed method of development of a sequential implementation resampling particle filter algorithm.

\section{SEQUENTIAL IMPLEMENTATION RESAMPLING PARTicle Filter}

The purpose of the particle filter in a geophysical application is to optimize the received data obtained from geophysical sensor since the received data is not consistent during data transmission. Despite that, the usage of basic particle filter did not solve the issue at all, because of the received data suffered from sample impoverishment phenomenon, which occurred during real time data transmission. In this paper, we aim to propose a new sequential implementation resampling particle filter with an adaptation of noise measurement and particle number (see figure 2). This study focuses on three situations of sample impoverishment, such as very low, low and high. These situations depends on real time data transmission setup. In figure 3 , it presents a block diagram showing the adaptive noise and particle based sequential implementation resampling algorithm. Initially, the system will receive the inertial data signal from the sensor. First, the noise measurement will be measured. If the noise measurement is high, the algorithm will switch to the roughening resampling algorithm. If the noise measurement is low, the particle number will be measured. Finally, the algorithm will carry out the resampling procedure based on modified resampling (if the particle is high) or variable resampling (if the particle is low). The following section will discuss the resampling algorithm that will be used together in this study. 


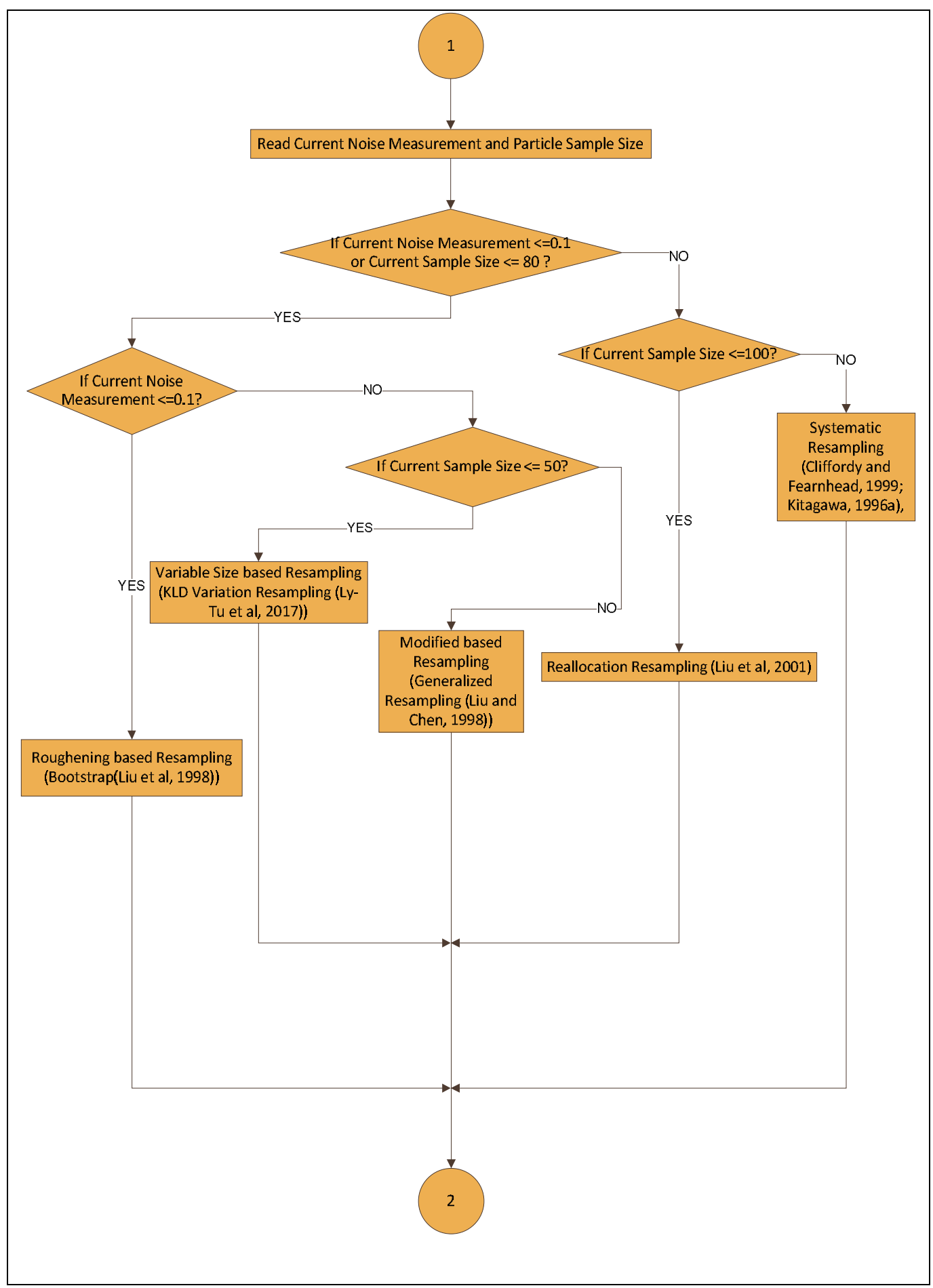

Figure 3: Architecture Block Diagram of Adaptive based Sequential Implementation Resampling Particle Filter (ASIRPF). (1 refer to start and 2 refer to end of the algorithm operation) 


\section{Single Distribution, Compound and SPecial STRATEGIES RESAMPLING}

This section discusses the resampling algorithm that will be used together in the sequential implementation resampling particle filter for countering the sample impoverishment phenomenon. The core design of this proposed algorithm is based on adaptation measurement of noise and particle sample size, then can be used to counter act the sample impoverishment in different situation. There are three types namely; single distribution resampling, compound resampling and special resampling [17][18][19][20] (The detail of the resampling schemes are described in further detail below. Firstly, single distribution resampling is a resampling scheme based on basic or straightforward resampling, and can be implemented in a single architecture. It can be the earliest example of fundamental resampling. Principally, it is used in particle filtering to prevent sample degeneracy, since it placed a computation burden on the system overall. There are currently two (2) general categories of single distribution resampling schemes; resampling based on normalized particle weights cumulative sum (RNPWC) and resampling based on residual (RB). The schemes differ based on normalised particle weights cumulative sum (RNPWC). It is using single resampling for each $j$ cycle, and resampling based on residual (RB) is using multiple resampling for each $j$ cycle. Secondly, compound resampling is a type of resampling scheme that relies on particle grouping, which is using predefined criteria applied before resampling. It is mainly used in particle filtering to reduce sample impoverishment, since it can place a computation burden to the system overall. It also decreases execution time and preserves particle diversity. One (1) of the unique characteristics of compound resampling is the resampling of the low weighted particle) this shows how compound resampling preserves particle diversity. Currently, there are three (3) general categories of compound resampling scheme; dynamic threshold based resampling, fixed threshold based resampling, and resampling that considers account particle values. Both dynamic threshold based resampling and fixed threshold based resampling function by resampling based on grouping predefined thresholds (the threshold can be dynamic or fixed) for weight function. The third (3rd) type is based on grouping a predefined threshold of particle value (or particle state). All compound resampling schemes will be described in greater detail in the following subsections [20]. Thirdly, is special strategies resampling, basically, it is referred to resampling schemes that focus on the modification of particle attributes, such as those of sample sizes, weights and state conditions. Since this resampling scheme was found to have resulted in various types of particle distribution, a number of special strategies in the likes of variable size based resampling, modified based resampling and roughening based resampling was therefore suggested. Briefly, these modified based resampling schemes function by modifying the particle weight distribution (also known as particle weight modification), meanwhile, the sample size of the particle distribution is altered by the variable size based resampling schemes. Under the variable size based resampling schemes, its computational complexity is reduced when a small number of particles are used on the different samples that were provided over time as according to the predefined condition. Apart from the improved tracking accuracy through the selection of a large particle number (or particle sample size modification), the roughening scheme had also modified the particle state by disrupting the resampled particles position as a way of reducing the impoverishment condition after the resampling process (also known as particle state modification). The following section will discuss the experimental setup of the method used in this study.

\section{Results}

The aim of this paper is to design a new sequential implementation resampling particle filter with an adaptation of noise measurement and particle number. This is due to previous algorithm cannot counter act the sample impoverishment phenomena in different situation. In this section, we discuss the experiment setup and the result of the proposed approach. This section discusses the experiment result of the proposed approach. There is two (2) part, the first part is the performance validation result, and the second part is a case study performance result. Currently, a number of simulations have been conducted in order to study the performance validation of the proposed particle filter algorithm. For details of these proposed particle filter algorithm, readers are referred to the pseudocodes given in the tutorial (the MATLAB codes for these resampling algorithms can be found at [26]). The simulation shows the state estimation errors of proposed particle filter algorithm were similar to those of the previously developed resampling schemes. 


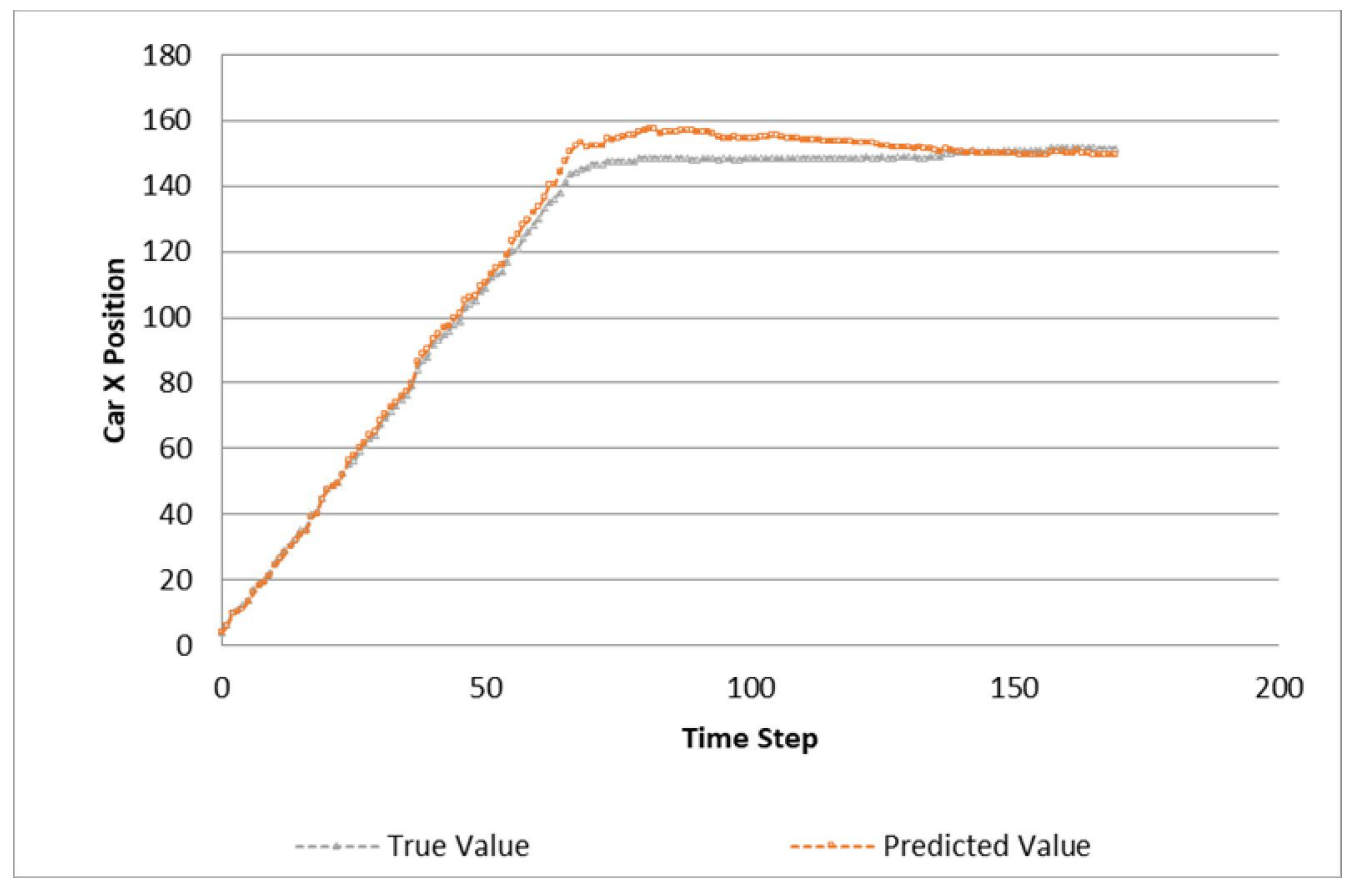

Figure 14: Predicted Value of X Position

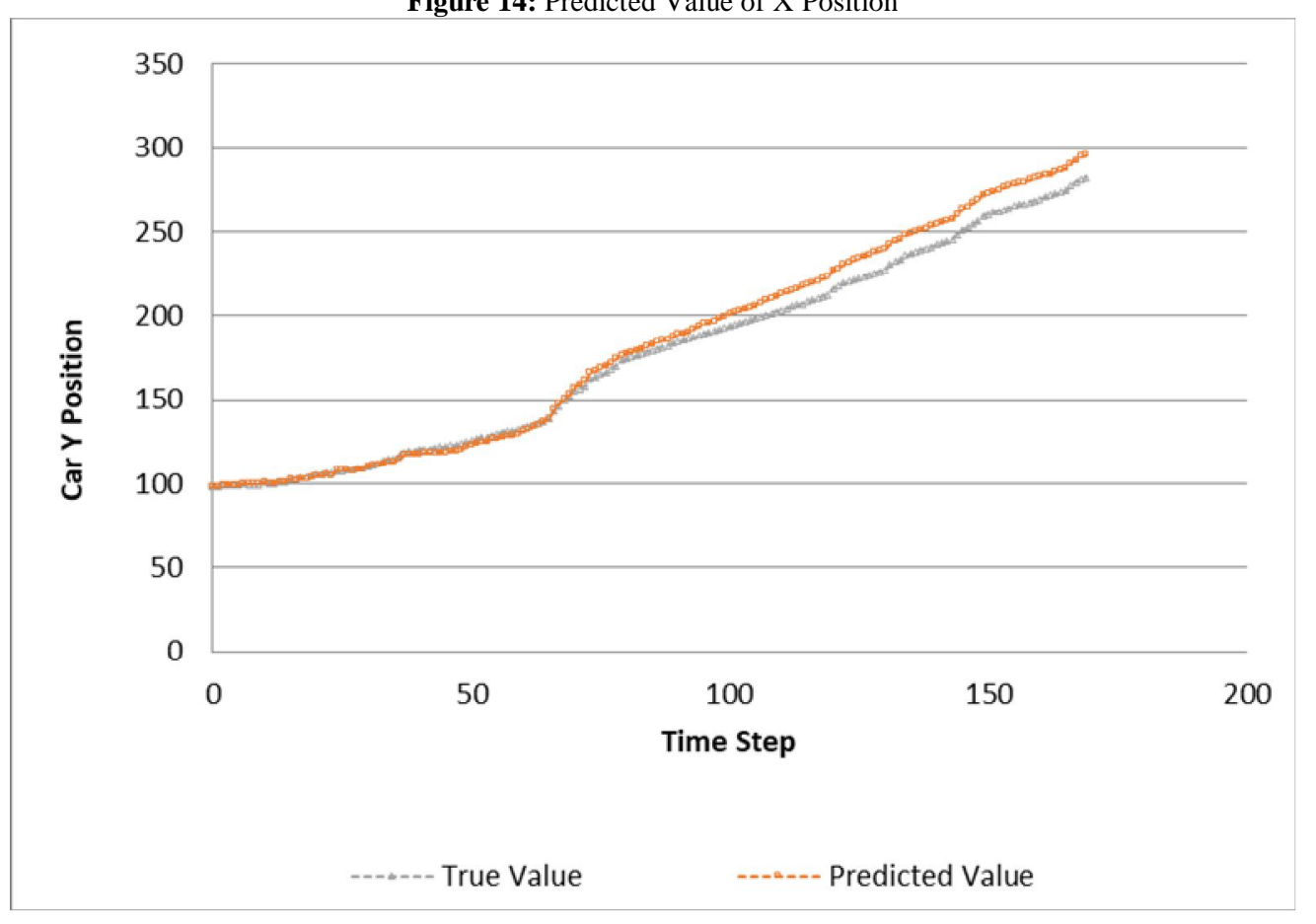

Figure 15: Predicted Value of Y Position 
This is because all of the resampling scheme have the same basic resampling scheme which eliminating low particle weight and replicating high particle weight. It is also indicating that the results for the proposed particle filter algorithm simulation are valid. In particular, the proposed algorithm can reduce the RMSE by $7.47 \%, 5.13 \%, 13.22 \%$, and $73.28 \%$ in comparison with single distribution resampling, compound 1 resampling, compound 2 resampling and special strategies resampling, respectively. This making the proposed algorithm achieved an average improvement of $24.78 \%$ compared to previous algorithm. Next result is showing the overall performance of proposed particle filtering when implemented in the case study. The case study is about the geophysical application during car moving (which from paper, [21] ). Basically, the digital road map is used to constrain the possible positions, where a dead reckoning of wheel speeds is the main external input to the algorithm. By matching the driven path to a road map, a vague initial position (order of kilometres) can be improved to a meter accuracy.

Generally, it can be seen the value prediction is a near to true value, but after time step of 50, the predicted value seems farther from the true value. This is because the nature of dead reckoning based positioning, which can generate the accumulated error. As a final result, the proposed particle filtering can achieve RMSE of $8.8857 \mathrm{~m}$. This is also shown the proposed particle filtering is suitable to be implemented in the geophysical application in the environment of the car moving by RMSE is less than $+-10 \mathrm{~m}$ which is better than RMSE of the conventional car navigation system. It is because the ASIRPF have the capability to do resampling in a different environment, specifically in different situation or stage of sample impoverishment. Even though the positioning determination was done in a moving environment (which is considered a challenging environment), it seems the ASIRPF can overcome the phenomenon. In addition, the proposed particle filtering (ASIRPF) is also useful in an inertial positioning system, which is can be used as a replacement or assisting of the global navigation satellite system.

\section{CONCLUSION AND FUTURE WORKS}

This paper describe about the optimization data in the geophysical applications, since the location determination of the geophysical application can be extremely challenging, if retrieved signal is unavailable or weak. In order to solve the issue, the adaptations towards to particle sample size and noise, must be made, to make the particle filter more intelligent, reliable and robust in a long time it is running. In this paper, a new algorithm of sequential implementation resampling particle filter by adapting the particle sample size and sensor noise measurement has been proposed. As the results, the paper shows the proposed solution can achieve an average improvement of $24.78 \%$ by reducing RMSE of state estimation compared to previous algorithm. In the future, it is expected to contribute to the modernization of geophysical applications. The results of this paper could be significantly contributing to modernizing the current real time geophysical applications and provide useful findings for use in other location positioning system [22][23][24][25] or other framework [27] [28] [29]. In the future, the researchers propose to continue experimenting by integrating other sensor such solar system in order to find more valuable findings.

\section{ACKNOWLEDGEMENT}

The research has been done in Universiti Teknologi Malaysia under the supervision of Assoc. Prof. Dr Mohd Murtadha Mohamad and Dr. Raja Zahilah Raja Mohd Radzi. The publication of this paper is funded by Universiti Teknikal Malaysia Melaka.

\section{REFERENCES}

1. Y. Li, Y. Zhuang, P. Zhang, H. Lan, X. Niu, and N. El-Sheimy, "An improved inertial/wifi/magnetic fusion structure for indoor navigation," Inf. Fusion, vol. 34, pp. 101-119, 2017.

2. W. M. Y. W. Bejuri, M. M. Mohamad, and R. Zahilah, "Optimization of Rao-Blackwellized Particle Filter in Activity Pedestrian Simultaneously Localization and Mapping (SLAM): An Initial Proposal," Int. J. Secur. Its Appl., vol. 9, no. 11, pp. 377-390, 2015.

3. W. M. Y. W. Bejuri, M. M. Mohamad, R. Zahilah, and R. M. Radzi, "Emergency Rescue Localization (ERL) using GPS, Wireless LAN and Camera," Int. J. Softw. Eng. Its Appl., vol. 9, no. 9, pp. 217-232, 2015.

4. W. M. Y. W. Bejuri, M. M. Mohamad, and R. Z. R. M. Radzi, "Offline Beacon Selection-Based RSSI Fingerprinting for Location-Aware Shopping Assistance: A Preliminary Result," in New Trends in Intelligent Information and Database Systems, vol. 598, Springer, 2015, pp. 303-312.

5. W. M. Y. W. Bejuri and M. M. Mohamad, "Performance Analysis of Grey-World-based Feature Detection and Matching for Mobile Positioning Systems," Sens. Imaging, vol. 15, no. 1, pp. 1-24, 2014.

6. P. Vadakkepat and L. Jing, "Improved particle filter in sensor fusion for tracking randomly moving object," IEEE Trans. Instrum. Meas., vol. 55, no. 5, pp. 1823-1832, 2006.

7. S.-H. Jung, G. Lee, and D. Han, "Methods and Tools to Construct a Global Indoor Positioning System," IEEE Trans. Syst. Man Cybern. Syst., 2017, Accessed: Feb. 19, 2017. [Online]. Available: http://ieeexplore.ieee.org/abstract/document/7805133/. 
8. X. Li, D. Wei, Q. Lai, Y. Xu, and H. Yuan, "Smartphone-based integrated PDR/GPS/Bluetooth pedestrian location," Adv. Space Res., vol. 59, no. 3, pp. 877-887, 2017.

9. Y. Li, Y. Zhuang, P. Zhang, H. Lan, X. Niu, and N. El-Sheimy, "An improved inertial/wifi/magnetic fusion structure for indoor navigation," Inf. Fusion, vol. 34, pp. 101-119, 2017.

10. Q. Liu, J. Qiu, and Y. Chen, "Research and development of indoor positioning," China Commun., vol. 13, no. Supplement2, pp. 67-79.

11. H. Ren, P. Chai, Y. Zhang, D. Xu, T. Xu, and X. Li, "Semiautomatic indoor positioning and navigation with mobile devices," Ann. GIS, pp. 1-12, 2017.

12. M. V. Albert, I. Shparii, and X. Zhao, "The Applicability of Inertial Motion Sensors for Locomotion and Posture," in Locomotion and Posture in Older Adults, Springer, 2017, pp. 417-426.

13. G. Retscher and F. Roth, "Wi-Fi Fingerprinting with Reduced Signal Strength Observations from Long-Time Measurements," in Progress in Location-Based Services 2016, Springer, 2017, pp. 3-25.

14. J. M. Pak, C. K. Ahn, Y. S. Shmaliy, P. Shi, and M. T. Lim, "Accurate and Reliable Human Localization Using Composite Particle/FIR Filtering," IEEE Trans. Hum.-Mach. Syst., 2016, Accessed: Feb. 16, 2017. [Online]. Available: http://ieeexplore.ieee.org/abstract/document/7588034/.

15. N. J. Gordon, D. J. Salmond, and A. F. Smith, "Novel approach to nonlinear/non-Gaussian Bayesian state estimation," in IEE Proceedings F-Radar and Signal Processing, 1993, vol. 140, pp. 107-113, Accessed: Nov. 12, 2016. [Online]. Available: http://ieeexplore.ieee.org/xpls/abs_all.jsp?arnumber=2 10672.

16. G. Falco, M. Pini, and G. Marucco, "Loose and Tight GNSS/INS Integrations: Comparison of Performance Assessed in Real Urban Scenarios," Sensors, vol. 17, no. 2, p. $255,2017$.

17. T. Li, G. Villarrubia, S. Sun, J. M. Corchado, and J. Bajo, "Resampling methods for particle filtering: identical distribution, a new method, and comparable study," Front. Inf. Technol. Electron. Eng., vol. 16, no. 11, pp. 969-984, 2015.

18. T. Li, S. Sun, and T. P. Sattar, "Adapting sample size in particle filters through KLD-resampling," Electron. Lett., vol. 49, no. 12, pp. 740-742, 2013.

19. T. Li, T. P. Sattar, and S. Sun, "Deterministic resampling: unbiased sampling to avoid sample impoverishment in particle filters," Signal Process., vol. 92, no. 7, pp. 1637-1645, 2012.

20. T. Li, M. Bolic, and P. M. Djuric, "Resampling methods for particle filtering: classification, implementation, and strategies," IEEE Signal Process. Mag., vol. 32, no. 3, pp. 70-86, 2015.

21. F. Gustafsson et al., "Particle filters for positioning, navigation, and tracking," IEEE Trans. Signal Process., vol. 50, no. 2, pp. 425-437, Feb. 2002, doi: 10.1109/78.978396.

22. Z. Li, J. Gao, J. Wang, and Y. Yao, "PPP/INS tightly coupled navigation using adaptive federated filter," GPS Solut., vol. 21, no. 1, pp. 137-148, 2017.

23. I. Belhajem, Y. B. Maissa, and A. Tamtaoui, "An Improved Robust Low Cost Approach for Real Time Vehicle Positioning in a Smart City," in Industrial Networks and Intelligent Systems, Springer, 2017, pp. 77-89.

24. A. Ghetti, L. Vittuari, and M. Zanzi, "A Satellite and Inertial Navigation Solution in Crises Management Operation for First Responder Application," in Advances in Science and Technology, 2017, vol. 101, pp. 158-167, Accessed: Feb. 19, 2017. [Online]. Available: http://www.scientific.net/AST.101.158.

26. 25. H. Xing, J. Li, B. Hou, Y. Zhang, and M. Guo, "Pedestrian Stride Length Estimation from IMU Measurements and ANN Based Algorithm," J. Sens., vol. 2017, 2017, Accessed: Feb. 19, 2017. [Online]. Available:

https://www.hindawi.com/journals/js/2017/6091261/ab s/.

27. R.T. Jadhav, and Vikram S.P. "A Real Time Hardware Co-Simulation of Linearization of Nonlinear Sensor using ANFIS Linearizer." International Journal of Advanced Trends in Computer Science and Engineering, Vol 9 (3), 2020, pp. 2550 - 2556

28. Alsharman, Nesreen, et al. " International Journal of Advanced Trends in Computer Science and Engineering, Vol 9 (3), 2020, pp. 2535 - 2541

29. W. H. W.Ishak, and F. M. Yamin. "Student Acceptance on Game to Support Teaching and Learning." International Journal of Advanced Trends in Computer Science and Engineering, Vol 9 (3), 2020, pp. 2517-2521 